\title{
Bright gamma-ray sources observed by DArk Matter Particle Explorer
}

\author{
Yun-Feng Liang ${ }^{* a}$, Kai-Kai Duan ${ }^{a b}$, Zhao-Qiang Shen ${ }^{a b}$, Zun-Lei $\mathbf{X u}^{a}$, and Simone \\ Garrappa $^{c}$ on behalf of the DAMPE collaboration \\ ${ }^{a}$ Key Laboratory of Dark Matter and Space Astronomy, Purple Mountain Observatory, Chinese \\ Academy of Sciences, Nanjing 210008, China \\ ${ }^{b}$ University of Chinese Academy of Sciences, Beijing, 100012, China \\ ${ }^{c}$ Universitá degli Studi di Perugia, INFN-Perugia, Italy \\ E-mail: liangyf@pmo.ac.cn, duankk@pmo.ac.cn
}

\begin{abstract}
DArk Matter Particle Explorer (DAMPE) is a high energy cosmic ray and gamma-ray telescope sensitive in energies from $\sim 1 \mathrm{GeV}$ to $\sim 10 \mathrm{TeV}$. After beyond one year's on-orbit operation, tens of sources including pulsars (PSR), active galaxy nuclei (AGN), supernova remnants (SNR) etc. have been identified in the DAMPE gamma-ray sky. Here we present the preliminary analysis results of DAMPE observation of some selected bright sources, including their fluxes, test statistic values, spectra and light curves.
\end{abstract}

35th International Cosmic Ray Conference - ICRC2017

10-20 July, 2017

Bexco, Busan, Korea

${ }^{*}$ Speaker. 


\section{Introduction}

DArk Matter Particle Explorer (DAMPE) is a high energy cosmic ray and gamma-ray telescope [1]. DAMPE is able to measure gamma-rays with unprecedented energy resolution, which can be as good as $<1.5 \%$ (68\% containment). The scientific goals of DAMPE include, (1) measuring cosmic-ray spectra with unprecedented energy resolution and energy reach, (2) studying high-energy gamma-ray astronomy and (3) detecting indirect dark matter signals.

Gamma-ray observation is crucial important to identify the physical origin (e.g., IC or hadron origin) of the astrophysical sources. With a high performance Plastic Scintillation array Detector (PSD) equipped at the top of DAMPE instrument (see [1] for details of DAMPE structure), DAMPE also has ability to detect gamma-ray photons. After about 1.5 years' on-orbit observations, DAMPE had observed tens of gamma-ray sources including PSR, AGN, SNR etc. In this report, we will present the preliminary analysis of DAMPE observation of some selected bright sources, mainly focus on their spectra and light curves.

\section{Data analysis and samples}

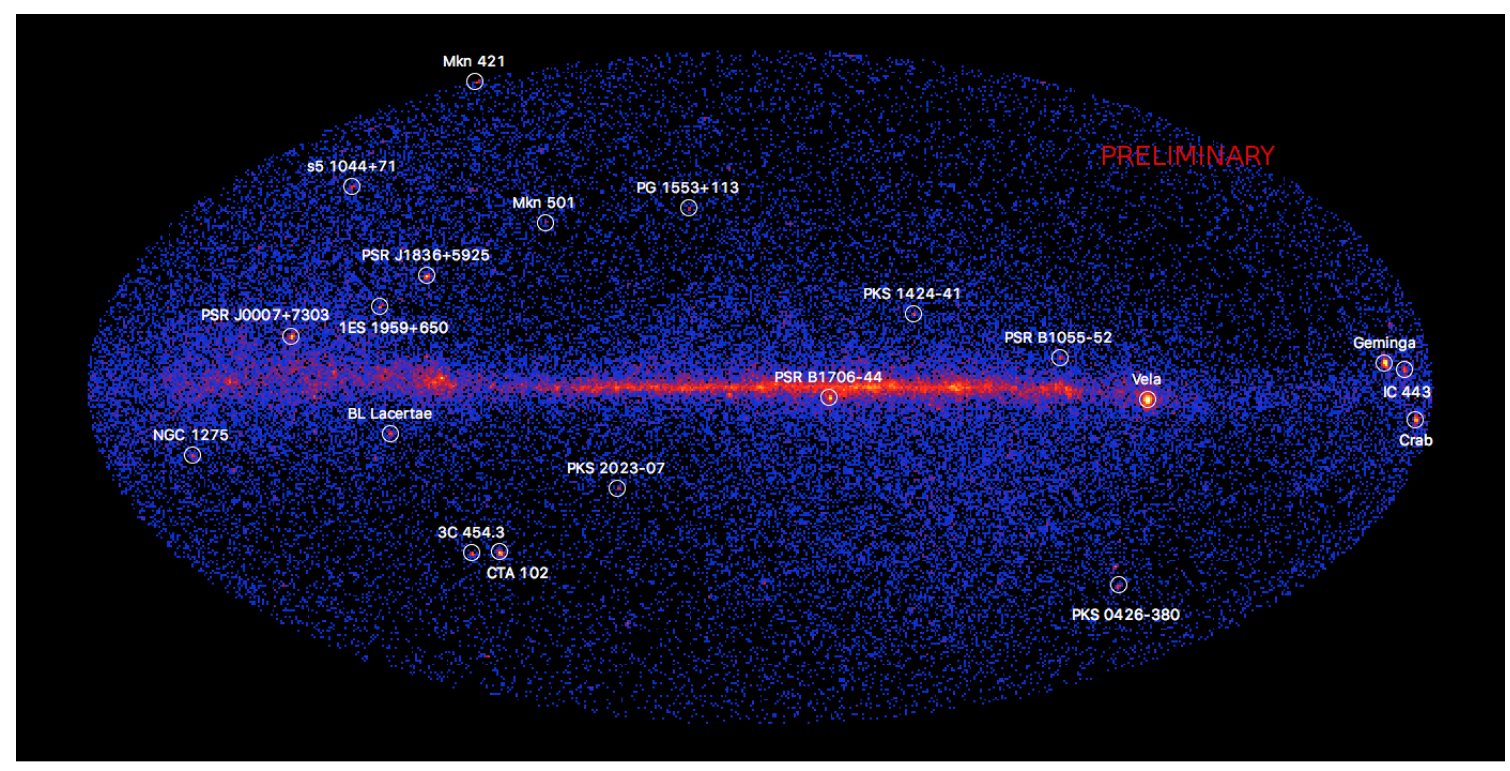

0.3

0.9

4.6

9.5

19.3

38.7

Figure 1: Here we show the locations of 20 selected bright DAMPE gamma-ray point sources in the sky over the 17 months' DAMPE all sky $>2 \mathrm{GeV}$ counts map. Note that the sky map is not corrected by exposure. The white circle around each source is a $2^{\circ}$ radius circle centered on its SIMBAD position.

We use a dedicated package named DmpST to perform our analysis. This package is developed to facilitate performing scientific analysis of DAMPE gamma-ray data and is based on the maximum likelihood algorithm [2]. With this package, we can do data selection, exposure calculation, convolving the model with DAMPE instrument response function (IRF), likelihood fitting and some other operations. The DAMPE IRFs, including effective area, point spread function and 
energy dispersion function, are derived based on pseudo-data generated in dedicated Monte Carlo detector simulation. For more detailed information of the DAMPE IRFs, one can see [3].

In this report, we take into account the data observed in the time range of 2016-01-01 to 201705-31 and in the energy range of 2 to $200 \mathrm{GeV}$. For each source of interest, we bin the data around the source into a $100 \times 100$ counts cube with spatial pixel size of $0.1^{\circ}$. The whole energy range we considered are subdivided into 10 energy bins equally in logarithmic space for the global fitting.

For the background point sources, we use the ones in the Fermi-LAT third point sources catalog (3FGL) [4] as seeds, and put them into our initial model file with their spectrum modified to power law function. After initial fitting, we remove the background point sources with $\mathrm{TS}<4$ in the DAMPE data. In current stage, due to both the facts that the amount of the data accumulated by DAMPE is not sufficient to generate a dedicated inter Galactic emission template and this component is theoretically instrument independent, we use the Fermi-LAT one in our analysis. For the isotropic diffuse component, which contain the contribution from extragalactic isotropic gammaray background emissions and residual cosmic-rays and thus is instrument dependent, we adopt a power law function to model its spectrum.

In this report, we select 20 point sources to show their results, which are among the most bright ones observed by DAMPE. Our samples include flat spectrum radio quasars (FSRQ), BL Lac objects (BL Lac), seyfert 2 galaxy (Seyf2 Gal), SNR and PSRs. In Figure 1, we demonstrate the locations of these selected sources in the sky together with the 17 months' DAMPE all sky $>2$ $\mathrm{GeV}$ counts map. Note that the sky map in Figure 1 is not corrected by exposure. The informations of these 20 bright sources are also listed in Table 1.

\section{Results}

With the analysis procedure described in the Section 2, we derived the preliminary analysis results of 20 bright sources. In the last three column of Table 1, we list the results obtained in the global fitting considering the data in the $2 \mathrm{GeV}$ to $200 \mathrm{GeV}$ energy range and the whole 17 months. As we can see, most of our selected sources have relative high test statistic (TS) value ${ }^{1}$, i.e. TS $>100$, indicating they are obviously observed by DAMPE. According to our analysis, the spectral indexes of these 20 sources range from about 1.7 to greater than 4 . The BL Lacs often have hard spectrum while the spectrum of FSRQs and PSRs are more softer, these characteristic are consistent with Fermi-LAT observation $[4,5]$ and theoretical predictions.

In Figure 2 and 3, we present the spectral energy distribution (SED) of these 20 bright DAMPE point sources. We subdivided the total energy range of $2 \mathrm{GeV}$ to $200 \mathrm{GeV}$ into 6 energy bin, the width of which are logarithmically equal. In each energy bin, we use the same likelihood analysis procedure described above to derive the flux of the source, but with the indexes of all sources fixed to their best fitted value obtained in the global fitting. The flux upper limit is derived if the TS value in that energy bin is smaller than 4. The dashed lines in Figure 2 and 3 are the best fitted power law spectrum in the global analysis.

For 12 AGNs in our samples, we also derived the aperture photometric light curves of them. For each time bin, the total number of observed photons within $2^{\circ}$ radius circle, $N_{i}$, is divided by

\footnotetext{
${ }^{1} \mathrm{TS}$ value is defined as TS $=-\ln \left(L_{\mathrm{nosrc}} / L_{\mathrm{withsrc}}\right)$, where $L_{\mathrm{nosrc}}$ and $L_{\text {withsrc }}$ denote the maximum poisson likelihood value obtained with target source included and not included in the model respectively.
} 
Table 1: 20 bright DAMPE gamma-ray sources concerned in this report.

\begin{tabular}{|c|c|c|c|c|c|c|c|}
\hline Source Name & 3FGL Name & Type & $\begin{array}{l}\text { RA } \\
\left({ }^{\circ}\right)\end{array}$ & $\begin{array}{c}\text { DEC } \\
\left({ }^{\circ}\right)\end{array}$ & $\begin{array}{c}\text { Flux }^{a} \\
\left(10^{-8} \mathrm{ph} / \mathrm{cm}^{2} / \mathrm{s}\right)\end{array}$ & Spectral Index & TS Value \\
\hline s5 1044+71 & 3FGL J1048.4+7144 & FSRQ & 162.12 & 71.74 & $1.102 \pm 0.186$ & $3.05 \pm 0.31$ & 196 \\
\hline $3 \mathrm{C} 454.3$ & 3FGL J2254.0+1608 & FSRQ & 343.50 & 16.15 & $4.563 \pm 0.603$ & $3.61 \pm 0.32$ & 385 \\
\hline CTA 102 & 3FGL J2232.5+1143 & FSRQ & 338.14 & 11.72 & $11.008 \pm 0.885$ & $2.65 \pm 0.12$ & 1330 \\
\hline Vela & 3FGL J0835.3-4510 & Pulsar & 128.84 & -45.18 & $52.630 \pm 1.520$ & $3.55 \pm 0.07$ & 7195 \\
\hline Geminga & 3FGL J0633.9+1746 & Pulsar & 98.48 & 17.77 & $33.058 \pm 1.385$ & $3.87 \pm 0.11$ & 4565 \\
\hline Crab & 3FGL J0534.5+2201 & Pulsar & 83.64 & 22.02 & $9.086 \pm 0.707$ & $2.60 \pm 0.12$ & 1067 \\
\hline Mkn501 & 3FGL J1653.9+3945 & BL Lac & 253.48 & 39.75 & $0.414 \pm 0.134$ & $2.00 \pm 0.36$ & 44 \\
\hline Mkn421 & 3FGL J1104.4+3812 & BL Lac & 166.12 & 38.21 & $2.165 \pm 0.317$ & $2.04 \pm 0.17$ & 331 \\
\hline IC443 & 3 FGL J0617.2+2234e & SNR & 94.31 & 22.58 & $3.659 \pm 0.517$ & $2.76 \pm 0.23$ & 211 \\
\hline PSR J1836+5925 & 3FGL J1836.2+5925 & Pulsar & 279.06 & 59.43 & $4.419 \pm 0.354$ & $3.88 \pm 0.22$ & 993 \\
\hline PSR J0007+7303 & 3FGL J0007.0+7302 & Pulsar & 1.77 & 73.05 & $3.459 \pm 0.305$ & $3.00 \pm 0.17$ & 716 \\
\hline PSR B1706-44 & 3FGL J1709.7-4429 & Pulsar & 257.43 & -44.49 & $8.246 \pm 0.652$ & $3.13 \pm 0.16$ & 729 \\
\hline PKS 0426-380 & 3FGL J0428.6-3756 & BL Lac & 67.17 & -37.94 & $1.739 \pm 0.306$ & $2.90 \pm 0.31$ & 200 \\
\hline NGC 1275 & 3FGL J0319.8+4130 & Seyf2 Gal & 49.96 & 41.51 & $1.669 \pm 0.273$ & $2.47 \pm 0.24$ & 192 \\
\hline PSR B1055-52 & 3FGL J1057.9-5227 & Pulsar & 164.50 & -52.46 & $1.339 \pm 0.237$ & $4.28 \pm 0.53$ & 120 \\
\hline PKS 1424-41 & 3FGL J1427.9-4206 & FSRQ & 216.99 & -42.11 & $1.943 \pm 0.306$ & $2.84 \pm 0.26$ & 214 \\
\hline PKS 2023-07 & 3FGL J2025.6-0736 & BL Lac & 306.42 & -7.60 & $2.141 \pm 0.411$ & $3.58 \pm 0.54$ & 149 \\
\hline BL Lacertae & 3FGL J2202.7+4217 & BL Lac & 330.69 & 42.28 & $2.003 \pm 0.316$ & $2.86 \pm 0.27$ & 213 \\
\hline 1ES $1959+650$ & 3FGL J2000.0+6509 & BL Lac & 300.02 & 65.15 & $1.095 \pm 0.154$ & $1.73 \pm 0.13$ & 335 \\
\hline PG $1553+113$ & 3FGL J1555.7+1111 & BL Lac & 238.94 & 11.19 & $1.206 \pm 0.257$ & $1.87 \pm 0.22$ & 147 \\
\hline
\end{tabular}

${ }^{a}$ Photon flux integrated in the energy range of $2 \mathrm{GeV}$ to $200 \mathrm{GeV}$.

corresponding effective exposure $\bar{\varepsilon}_{i}$ to derived the photon flux, here $i$ means in the $i$-th time bin. The $\bar{\varepsilon}_{i}$ is derived using the spectrum of the target source, $F(E)$, to weight the DAMPE exposure $\varepsilon(E)$ at the direction of that source,

$$
\bar{\varepsilon}_{i}=\frac{\int F(E) \varepsilon(E) \mathrm{d} E}{\int F(E) \mathrm{d} E} .
$$

For $F(E)$, we use the best fitted spectrum obtained in the global likelihood fitting. Figure 4 demonstrates the aperture photometric results. In Figure 4, the time bin size of the light curves is 30 days. The horizontal dashed line indicates the average flux obtained in the above global likelihood analysis. The shaded range represents the time bin in which the target source is not within the field of view of DAMPE. As is shown in Figure 4, among our 12 AGN samples, CTA 102 show obvious enhancement of its photon flux in the period of from the last several months of 2016 to the first several months of 2017, indicating this source is within a flare phase. This flare behavior is also observed by many other ground and space based telescopes [6, 7, 8]. Besides CTA 102, some other sources, e.g., s5 1044+71, 3C 454.3, PKS 2023-07, also show indications of variability.

\section{Summary}

In this report, we present our preliminary analysis results of 20 DAMPE gamma-ray point sources, which are among the most bright ones observed by DAMPE. With a dedicated analysis package, we derived the TS value, power law spectral index, spectral energy distribution and aperture photometric light of each source. Our results show that all these sources are obviously observed 

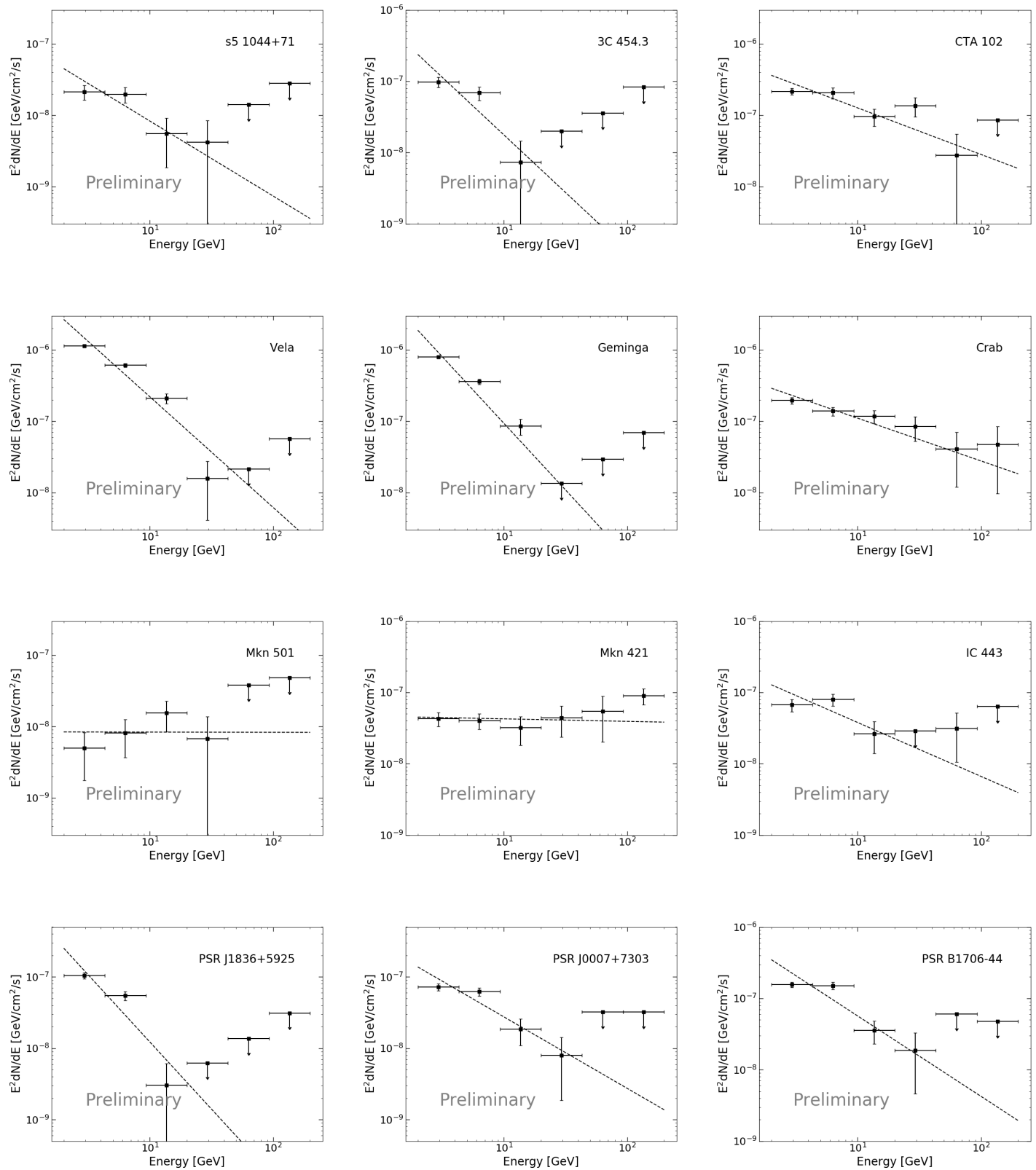

Figure 2: The SED of 20 selected bright DAMPE point sources. The flux upper limits are calculated if the TS values are smaller than 4 . The dashed lines represent the best fitted power law spectrum in the global analysis.

by DAMPE and their spectral properties are consistent with other observation and with theoretical prediction, revealing the good ability of DAMPE to study high-energy gamma-ray astronomy.

\section{Acknowledgments}

The DAMPE mission is funded by the strategic priority science and technology projects in 

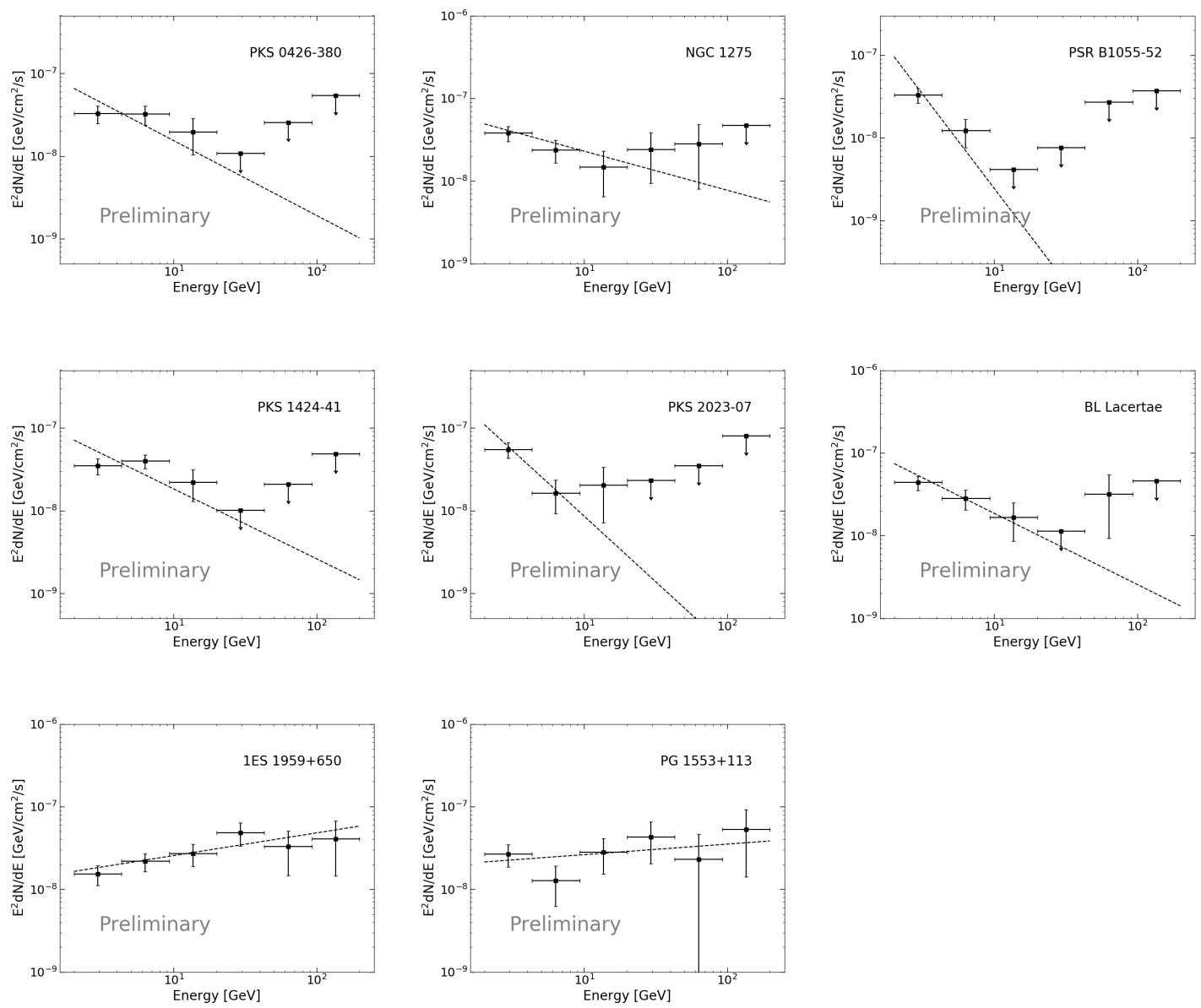

Figure 3: Continued.

space science of Chinese Academy of Sciences. In China the data analysis was supported in part by the National Key Research and Development Program of China (No. 2016YFA0400200), National Basic Research Program of China (No. 2013CB837000), NSFC (Nos. 11525313 and 11622327), and the 100 Talents Program of Chinese Academy of Sciences. In Europe the activities and the data analysis are supported by the Swiss National Science Foundation (SNSF), Switzerland; the National Institute for Nuclear Physics (INFN), Italy.

\section{References}

[1] J. Chang et al. (DAMPE Collaboration), Astropart. Phys. submitted, arXiv:1706.08453.

[2] J. R. Mattox, D. L. Bertsch et al., Astrophys. J. 461, 396 (1996).

[3] K. K. Duan et al., ICRC Proceeding, in preparation.

[4] F. Acero et al. (Fermi LAT Collaboration), Astrophys. J. Suppl. Ser. 218, 23 (2015).

[5] M. Ackermann et al. (Fermi LAT Collaboration), Astrophys. J. 810, 14 (2015)

[6] G. Minervini, A. Bulgarelli, C. Pittori et al., ATel, No. 10386, 386 (2017) 

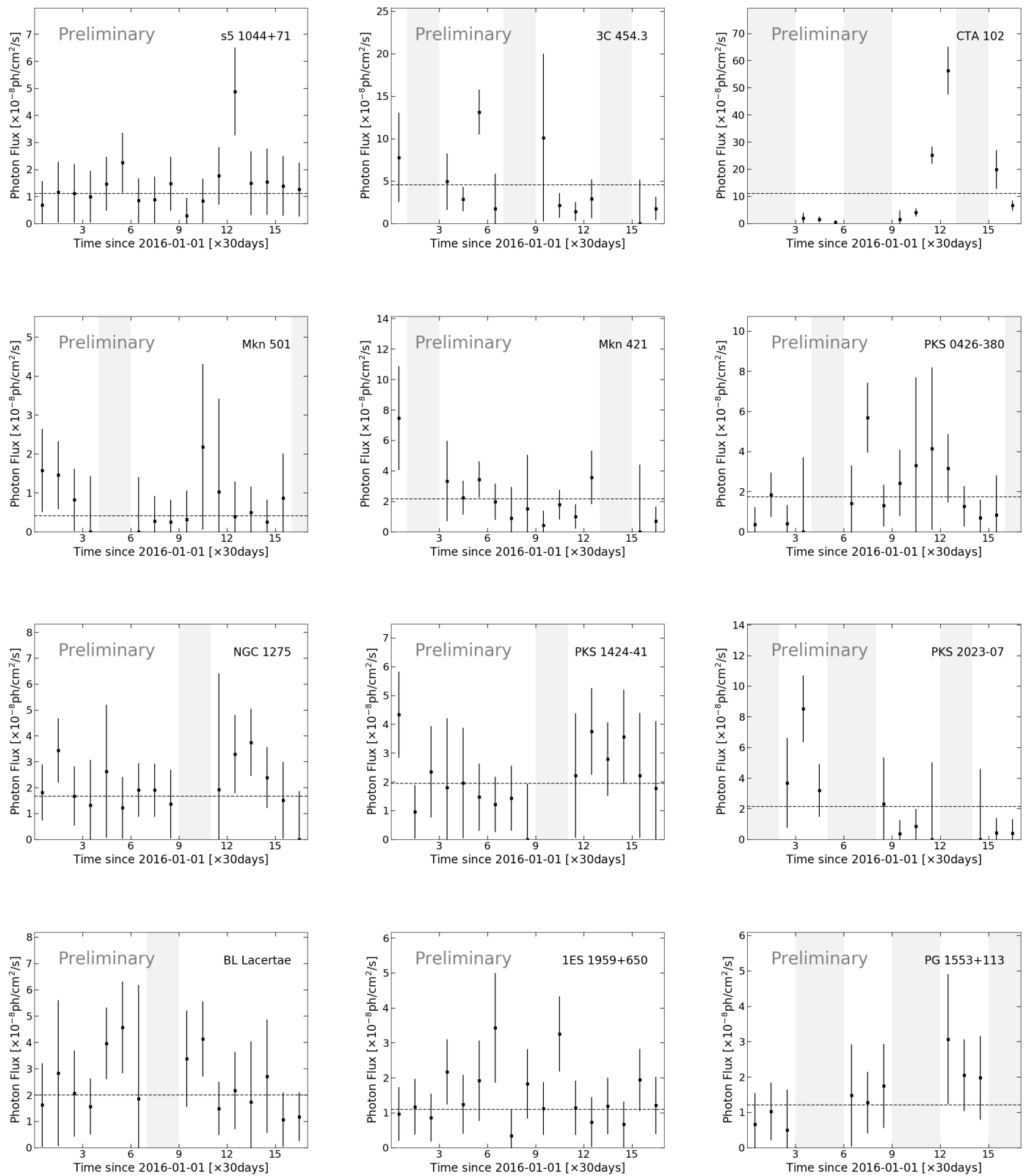

Figure 4: Aperture photometric light curves of 12 AGNs in our samples. The time bin size is 30 days. The horizontal dashed line indicates the average flux obtained in global likelihood analysis. The shaded range represents the time bin in which the target source is not within the field of view of DAMPE.

[7] S. Ciprini, ATel, No. 10292, 292 (2017)

[8] R. Ojha, B. Carpenter, and F. D’Ammand, ATel, 9924 (2017) 